\title{
FORECASTING CLOSING RETURNS OF BORSA ISTANBUL INDEX WITH MARKOV CHAIN PROCESS OF THE FUZZY STATES
}

\author{
DOI: 10.17261/Pressacademia.2017.362 \\ JEFA-V.4-ISS.1-2017(2)-p.15-24
}

\section{Ersin Kiral ${ }^{1}$, Berna Uzun ${ }^{2}$}

${ }^{1}$ Çukurova University. Department of Econometrics, Adana, 01330, Turkey. ekiral@cu.edu.tr

${ }^{2}$ Near East University. Department of Mathematics, , 99138. Nicosia, TRNC Mersin 10, Turkey. berna.uzun@neu.edu.tr

\section{To cite this document}

Kiral, E. and B. Uzun, (2017). Forecasting closing returns of Borsa Istanbul Index with Markov Chain Process of the fuzzy states. Journal of Economics, Finance and Accounting (JEFA), V.4, Iss.1, p.15-24.

Permemant link to this document: http://doi.org/10.17261/Pressacademia.2017.362

Copyright: Published by PressAcademia and limited licenced re-use rights only.

\section{ABSTRACT}

Purpose- The estimation regarding to the exact daily price of the stock market index has always been a difficult task in the business sector. Therefore, there are numerous research studies carried out to predict the direction of stock price index movement.

Methodology- Classical Markov chain model (MC) is commonly used for this prediction and it gives valuable signals about the movements of the closing returns of the stock market index. In this paper, we propose Markov Chain Model with Fuzzy States (MCFS) to predict the closing returns of Borsa Istanbul (BIST 100) index using triangular fuzzy numbers. We apply this method to hold the information while system moves between the extreme values of the states.

Findings- With this study, we show that the use of MCFS for the selected period provides a higher forecasting accuracy to the investors compared to MC model.

Conclusion- Markov chains of the fuzzy states defines a stochastic system more precisely than the classical Markov chains and it gives more sensitive future prediction opportunities. It can be used for estimating returns of individual common stocks and also for the other investment instruments.
\end{abstract}

Keywords: Stock return, fuzzy sets, conditional probability, Markov Decision Process, Markov Chain with fuzzy states JEL Codes: C44, C53, C61

\section{INTRODUCTION}

Decision Making has become the most important field for scientific, social and economic researchers. In a real world situations, decisions are made under the uncertain conditions and also in most applications we obtain the data by estimation or the experience which are imprecise and fuzzy. Therefore, decision- making problems can be described with fuzzy set theory more exactly than the classical crisp decision making theories.

Stock Index, which is related to the changes in the stock prices, plays a significant role in the business sector for the performance valuation. Many factors might have an effect on the stock market index such as the political events, general economic conditions, trader's expectations etc. Stock index prediction has been very interesting research topic for many years. Due to it's complex, dynamic and highly non-linear data over time, it is a very difficult task to predict the exact daily price of the stock market index. The direction of the stock market index correlates with the movement of the price index. Estimating the direction significantly influences the decision of the financial traders about buying or selling an instrument. Hence, the stock index prediction can provide investors to gain profit in the stock exchange. 
This paper is organized as follows: we provide literature rewiew and concept of the paper in Section 2. In Section 3, the data set, proposed methodology which is related to the fuzzy sets and the Markov chain process of the fuzzy sets are given. In Section 4, findings and discussions about the stock return estimation with both MCFS and MC models are presented. Our conclusions are given in Section 5.

\section{LITERATURE REVIEW}

Recently, various estimating models have been presented and applied for the stock market analysis. Hidden Markov Model (Rabiner \& Juang, 1993; Rabiner, 1993), widely implemented estimating models to estimate stock market data. Box and Jenkins (1976), used the Time series analysis to estimate and control. White $(1988,1989)$ used Neural Networks to estimate stock market of IBM daily stock returns. Henry (1993), used ARIMA model to predict the daily close and morning open price. But these conventional methods are not useful when non linearity exists in time series. Chiang, Urban and Baldridge, (1996), have estimated the end-of-year net asset value of mutual funds via ANN model. Kim and Han (2000), showed there are complex dimensionality and buried noise at the stock market data so that makes it hard to reestimate the ANN parameters. Romahi and Shen (2000) showed that, ANN sometimes suffers from over fitting problem. They have evolved rule, which depends on expert system and generated a method to predict financial market behaviour. Later on, hybridization models have been used for estimating financial behaviour. All of these methods has required expert knowledge to deal with the aforementioned problems. Hassan and Nath (2005), used HMM for optimizing the system in a better way. Hassan, Nath and Kirley (2006), integrated HMM and fuzzy logic rules to evolve the prediction accuracy on nonstationary stock data sets. Following this, Hassan, Nath and Kirley (2007), presented a fusion model of HMM, ANN and GA to estimate stock market. Badge (2012) used technical indicators as an input variable instead of stock prices for analysis. Gubta and Dhingra (2012) considered the fractional change in stock value and the intra-day high and low values of the stock to train the continuous HMM.

In previous studies, several research work had been applied to different methods and algorithms for training the model to estimate the next day close value of the stock market, by considering randomly generated Transition Probability Matrix (TPM), Emission Probability Matrix (EPM) and prior probability matrix.

Markov process is a stochastic model that has the Markov property. It can be used to model a random system, which changes its states according to the transition rule, which depends on the current state. Markov decision processes (MDPs) give mathematical framework for modeling the situations where outcomes are partly random and partly under the control of a decision maker. MDPs are commonly used for a wide range of optimization problems which are solved with dynamic programming and reinforcement learning. MDPs were known at least as early as the 1950s (Bellman, 1957). This method used in a wide range of disciplines, such as biology, medicine, social science, robotics, automated control, economics, and manufacturing. It has been studied well in the literature but this method relies on the states with sharp bounds which lead to counting the movements between extreme values of the states as the movements between average values of the states.

In order to describe unclear situations mathematically, Zadeh defined fuzzy sets in 1965. After Zadeh's work, Thomason (1977), introduced fuzzy matrix for the first time and Kruce, Buck-Emden and Cordes (1987) introduced the fuzzy Markov chain as a classical Markov chain based on fuzzy probabilities and used fuzzy sets to generate transition matrix of the uncertain datas in the Markov chain. Fuzzy Markov chain is demonstrated as the concept of fuzzy relation and its compositions (Sanchez, 1976). It can be used while the decision maker prefers subjective probabilities to model the uncertainties (Vajargah \& Gharehdaghi, 2012). Yoshida (1994) generated a Markov fuzzy model with a transition possibility measures. Stow'ınski (1998), showed that we can use a fuzzy set representation in order to deal with uncertain data and flexible requirements. Avrachenkov and Sanchez (2000) analyzed fuzzy Markov chains and its properties in detail. Symeonaki and Stamou (2004), generated theory of the Markov system with fuzzy states and analyzed properties of the Markov model for uncertain boundaries. Kuranoa, Yasuda, Jakagami and Yoshida (2006), used fuzzy states to show fuzzy transition probabibilities. Salzenstein, Collet, Lecam and Hatt (2007), generated the dynamic fuzzy Markov chain model and compared with the static fuzzy Markov chain model. Pardo and Fuente (2010) used Markovian decision processes with fuzzy states to calculate the best policy to be implemented regarding publicity decisions in a queueing system. Zhou, Tang, Xie, Li and Zhang (2013) used fuzzy probability-based Markov chain model to estimate regional long-term electric power demand.

In order to deal with uncertain and complex nature of the stock market index movements we used MCFS to predict the movements of the closing returns of the BIST 100 index. For this aim, we have categorised closing returns for the BIST 100 index datas as the 21 fuzzy states with the triangular fuzzy sets. Then we obtained Markov transition matrix of the fuzzy states. Finally, we have estimated next day closing return of the BIST 100 index by using Markov transition matrix of the fuzzy states and partition degrees of the present session state which is choosen randomly. 


\section{DATA AND METHODOLOGY}

\subsection{Sample}

The sample data cover approximately 3325 closing returns of the BIST 100 index between the period of Jan.02, 2003 and Mar.10, 2016. The data were obtained from the electronic data delivery system of the Borsa Istanbul.

The closing returns $R_{t}$ were calculated as a percentage change of the BIST 100 index closing values $P_{t} ; R_{t}=\left(P_{t}-\right.$ $\left.P_{t-1}\right) / P_{t-1}$, where $t$ denotes the sessions $(t=2,3, \ldots, 3325)$. The average return $\mu_{R}$ is approximately $0,08 \%$ and the standard deviation is $1,8 \%$ for the given period. The standard deviation is approximately 22 times higher than the expected return. Therefore the BIST 100 index return involves significant risk for the investors.

\subsection{Methodology}

\section{An overview of the fuzzy sets}

Here, we give some necessary definitions about fuzzy set theory.

Definition 1: A fuzzy set $\tilde{A}$ in $I R$ is a set of ordered pairs:

$$
\tilde{\mathrm{A}}=\left\{\left(x, \mu_{\tilde{\mathrm{A}}}(x)\right) \mid x \in I R\right\}
$$

where $\mu_{\tilde{\AA}}: I R \rightarrow[0,1]$ and $\mu_{\tilde{\AA}}(x)$ is called the membership function for the fuzzy set.

Definition 2: A fuzzy number is a fuzzy set on the real line that satisfies the conditions of normality and convexity.

Definition 3: A fuzzy number that represented with three points as follows: $\tilde{\mathrm{A}}=\left(a_{1}, a_{2}, a_{3}\right),\left(a_{1}<a_{2}<a_{3}\right)$ and whose membership function is given by

$$
\mu_{\tilde{\AA}}(x)=\left\{\begin{array}{cr}
\frac{x-a_{1}}{a_{2}-a_{1}}, & \text { if } a_{1} \leq x \leq a_{2} \\
\frac{a_{3}-x}{a_{3}-a_{2}}, & \text { if } a_{2} \leq x \leq a_{3} \\
0, & \text { otherwise }
\end{array}\right.
$$

is called a triangular fuzzy number.

\section{Probabilities of Markov chains}

Let $X_{t}$ be the state of the system at time $t$. We assume that the transition probability matrix of a finite state Markov chain $P$ is;

$$
P=\left[p_{i j}\right] \forall i, j=\{0,1, \ldots, N\},
$$

where $p_{i j}$ denotes the transition probability from state $i$ to state $j$ (of one step) and $p_{i j} \geq 0$ and for $\forall i, j$ :

$$
p_{i j}=P\left\{X_{t+1}=j \mid X_{t}=i\right\}=P\left\{X_{1}=j \mid X_{0}=i\right\},
$$

with $\sum_{j=0}^{N} p_{i j}=1$. And the transition probability from state $i$ to state $j$ in $r$ steps $p_{i j}^{r}$ is:

$$
\begin{gathered}
P^{r}=\left[p_{i j}^{r}\right] p_{i j}^{r} \geq 0, \quad \forall i, j \in\{0,1, \ldots, N\}, \\
p_{i j}^{r}=P\left\{X_{t+r}=j \mid X_{t}=i\right\}=P\left\{X_{r}=j \mid X_{0}=i\right\} .
\end{gathered}
$$

And

$$
P^{r}=(P)^{r}
$$




\section{Markov chain process with fuzzy states}

It is very useful to define the problem with fuzzy states if there is not sufficient information about the system and also when the states of the system are known, but the number of the states are too large to make a decision (Pardo \& Fuente, 2010).

The most important difference between MC and MCFS depends on the values assumed for the variables. While in MCFS those values denote the fuzzy membership degrees, in MC they denote the probabilities. As a result, MCFS contains a possibilistic modeling of the problem, which is more flexible than the classical probabilistic approach with crisp states.

\section{Probabilities of Markov chain with fuzzy states}

Let $X=\left\{x_{1}, x_{2}, \ldots, x_{n}\right\}$ be a given set. A fuzzy partition of $X$ is a family of fuzzy subset of $X$, denoted by $A=\left\{\tilde{\mathrm{A}}_{1}\right.$, $\left.\tilde{\mathrm{A}}_{2}, \ldots, \tilde{\mathrm{A}}_{N}\right\},\left(\forall i=\{1,2, \ldots, N\}, \quad \tilde{\mathrm{A}}_{i} \neq \emptyset\right.$ and $\left.\tilde{\mathrm{A}}_{i} \neq X\right)$ with the corresponding membership functions $\mu_{\tilde{\mathrm{A}}_{1}}, \mu_{\tilde{\mathrm{A}}_{2}}, \ldots, \mu_{\tilde{\mathrm{A}}_{N}}$ which satisfy the following condition;

$$
\sum_{i=1}^{N} \mu_{\tilde{\AA}_{i}}\left(x_{r}\right)=1
$$

$\forall x_{r} \in X$, and $r=\{1, \ldots, n\}$.

The notion of fuzzy partition is used to define the fuzzy states for the Markovian decision process.

Let $\left\{\tilde{\mathrm{A}}_{1}, \tilde{\mathrm{A}}_{2}, \ldots, \tilde{\mathrm{A}}_{N}\right\}$ be a set of fuzzy states, as each fuzzy subset $\tilde{\mathrm{A}}_{i}, i \in\{1, \ldots \ldots, n\}$ denotes a fuzzy state in the initial Markov chain.

Definition 4: The probability of fuzzy initial state $P\left(\tilde{\mathrm{A}}_{i}\right)=P\left(X_{0}=\tilde{\mathrm{A}}_{i}\right)$ is defined by using the probability of fuzzy event:

$$
P\left(\tilde{\mathrm{A}}_{i}\right)=P\left\{\tilde{X}_{0}=\tilde{\mathrm{A}}_{i}\right\}=\sum_{s=0}^{N} P\left\{X_{0}=s\right\} \mu_{\tilde{\AA}_{i}}(s)
$$

Definition 5: (Pardo \& Fuente, 2010), The conditional probability of the fuzzy state $\tilde{\mathrm{A}}_{j}$, given the initial state $m$, with $j \in\{1, \ldots, n\}$ and $m \in\{0, \ldots, N\}$, is:

$$
P\left(\tilde{\mathrm{A}}_{j} \mid m\right)=P\left\{\tilde{X}_{1}=\tilde{\mathrm{A}}_{j} \mid X_{0}=m\right\}=\sum_{s=0}^{N} P\left\{\tilde{X}_{1}=\tilde{\mathrm{A}}_{j} \mid X_{0}=m\right\} \mu_{\tilde{\mathrm{A}}_{j}}(s)
$$

and represents the transition probability to fuzzy state (of one step).

Proposition 1: (Pardo \& Fuente, 2010), The conditional probability of the fuzzy event $\tilde{\mathrm{A}}_{j}$ given the fuzzy event $\tilde{\mathrm{A}}_{i}, i, j \in$ $\{1, \ldots, n\}$ is a function of a linear combination of probabilities $P\left(\tilde{\mathrm{A}}_{j} \mid m\right), m \in\{0, \ldots, N\}$ as in equation:

$$
P\left(\tilde{\mathrm{A}}_{j} \mid \tilde{\mathrm{A}}_{i}\right)=P\left\{\tilde{X}_{1}=\tilde{\mathrm{A}}_{j} \mid \tilde{X}_{0}=\tilde{\mathrm{A}}_{\mathrm{i}}\right\}=\sum_{m=0}^{N} P\left(\tilde{\mathrm{A}}_{j} \mid m\right) \frac{P\left\{X_{0}=m\right\} \mu_{\tilde{\mathrm{A}}_{i}}(m)}{P\left(\tilde{\mathrm{A}}_{i}\right)}
$$

and represents the probability of transition from the fuzzy initial state to the fuzzy final state (of one step).

The transition probability matrix of fuzzy states can be calculated by the equation (6).

\subsection{Methodology for Markov Chain Model}

Closing returns of the BIST 100 index are transformed into 21 discrete categorical states from high loss $S_{-10}$ to the positive high return $S_{10}$ according to functions below. For this aim, we defined the $k$ integer numbers which is based on $R_{t}$ as $k-1<\frac{R_{t}+0.12 \%}{0.24 \%} \leq k$ where $-2,28 \%<R_{t} \leq 2.28 \%$ and the $k$-th Markov states for $k \in\{-9, \ldots, 9\}$ as followings:

$$
\begin{aligned}
& S_{10}=\left\{\begin{array}{lr}
1, & \text { if } R_{t}>2.28 \% \\
0, & \text { otherwise }
\end{array}\right. \\
& S_{k}= \begin{cases}1, & \text { if }(2 k-1) 0.12 \%<R_{t} \leq(2 k+1) 0.12 \% \\
0, & \text { otherwise }\end{cases} \\
& S_{-10}= \begin{cases}1, & \text { if } R_{t} \leq-2.28 \% \\
0, & \text { otherwise }\end{cases}
\end{aligned}
$$




\section{Table 1: Transformed States of the Closing Returns for Some Days}

\begin{tabular}{|c|c|c|c|c|c|c|c|c|c|c|c|c|c|c|c|c|c|c|c|c|c|c|}
\hline Date & $R_{t}$ & $S_{-10}$ & $S_{-9}$ & $S_{-8}$ & $S_{-7}$ & $S_{-6}$ & $S_{-5}$ & $S_{-4}$ & $S_{-3}$ & $S_{-2}$ & $S_{-1}$ & $S_{0}$ & $S_{1}$ & $S_{2}$ & $S_{3}$ & $S_{4}$ & $S_{5}$ & $S_{6}$ & $S_{7}$ & $S_{8}$ & $S_{9}$ & $S_{10}$ \\
\hline Mar.10, 2016 & $0.36 \%$ & 0 & 0 & 0 & 0 & 0 & 0 & 0 & 0 & 0 & 0 & 0 & 1 & 0 & 0 & 0 & 0 & 0 & 0 & 0 & 0 & 0 \\
\hline Mar.09, 2016 & $1.36 \%$ & 0 & 0 & 0 & 0 & 0 & 0 & 0 & 0 & 0 & 0 & 0 & 0 & 0 & 0 & 0 & 0 & 1 & 0 & 0 & 0 & 0 \\
\hline Mar.08, 2016 & $0.26 \%$ & 0 & 0 & 0 & 0 & 0 & 0 & 0 & 0 & 0 & 0 & 0 & 1 & 0 & 0 & 0 & 0 & 0 & 0 & 0 & 0 & 0 \\
\hline Mar.07, 2016 & $0.38 \%$ & 0 & 0 & 0 & 0 & 0 & 0 & 0 & 0 & 0 & 0 & 0 & 0 & 1 & 0 & 0 & 0 & 0 & 0 & 0 & 0 & 0 \\
\hline Mar.06, 2016 & $0.47 \%$ & 0 & 0 & 0 & 0 & 0 & 0 & 0 & 0 & 0 & 0 & 0 & 0 & 1 & 0 & 0 & 0 & 0 & 0 & 0 & 0 & 0 \\
\hline Mar.03, 2016 & $0.05 \%$ & 0 & 0 & 0 & 0 & 0 & 0 & 0 & 0 & 0 & 0 & 1 & 0 & 0 & 0 & 0 & 0 & 0 & 0 & 0 & 0 & 0 \\
\hline Mar.02, 2016 & $1.10 \%$ & 0 & 0 & 0 & 0 & 0 & 0 & 0 & 0 & 0 & 0 & 0 & 0 & 0 & 0 & 0 & 1 & 0 & 0 & 0 & 0 & 0 \\
\hline Mar.01, 2016 & $0.19 \%$ & 0 & 0 & 0 & 0 & 0 & 0 & 0 & 0 & 0 & 0 & 0 & 1 & 0 & 0 & 0 & 0 & 0 & 0 & 0 & 0 & 0 \\
\hline Feb.29, 2016 & $1.18 \%$ & 0 & 0 & 0 & 0 & 0 & 0 & 0 & 0 & 0 & 0 & 0 & 0 & 0 & 0 & 0 & 1 & 0 & 0 & 0 & 0 & 0 \\
\hline Feb.26, 2016 & $-0.36 \%$ & 0 & 0 & 0 & 0 & 0 & 0 & 0 & 0 & 1 & 0 & 0 & 0 & 0 & 0 & 0 & 0 & 0 & 0 & 0 & 0 & 0 \\
\hline Feb.25, 2016 & $1.53 \%$ & 0 & 0 & 0 & 0 & 0 & 0 & 0 & 0 & 0 & 0 & 0 & 0 & 0 & 0 & 0 & 0 & 1 & 0 & 0 & 0 & 0 \\
\hline Feb.24, 2016 & $-1.90 \%$ & 0 & 0 & 1 & 0 & 0 & 0 & 0 & 0 & 0 & 0 & 0 & 0 & 0 & 0 & 0 & 0 & 0 & 0 & 0 & 0 & 0 \\
\hline
\end{tabular}

As it is shown in Table 1, we transformed 3325 closing returns of BIST 100 index to the defined 21 discrete states. Then, we calculated all transitions numbers of states from the present session to the next session for the period considered. We also used conditional probabilities of the Markov chain to obtain one step transition probability matrix which is shown in Table 2 .

\section{Table 2: One Step Conditional Probability Matrix}

\begin{tabular}{|c|c|c|c|c|c|c|c|c|c|c|c|c|c|c|c|c|c|c|c|c|c|}
\hline & & & & & & & & & & & & & & & & & & & & & \\
\hline$S_{-10}$ & 17 & .03 & .03 & .04 & 03 & & & & & & & & & & & & & & & & \\
\hline S & .13 & .02 & .08 & .04 & .13 & 4 & 02 & & 00 & .04 & & & & & & & & & & & \\
\hline 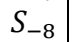 & & .00 & 1 & .03 & 7 & & 03 & & 5 & .07 & J & & & & & & & & & & \\
\hline & & 01 & 12 & .00 & ) & & $\Omega$ & & & & & & & & & & & & & & \\
\hline & & & & 55 & & & & & & & & & & & & & & & & & \\
\hline & & .02 & 2 & .03 & & & 0 & & 06 & & o & & & & & & & & & & 12 \\
\hline & & .03 & 2 & .03 & & & 0 & . & .08 & .0 & .08 & & 0 & & & & & & & & $\partial \epsilon$ \\
\hline 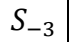 & & .02 & 4 & .01 & U. & & .0 & & .04 & .0 & .1 & & & & & & & & & & .07 \\
\hline & .0 & .00 & 3 & .03 & & & 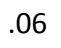 & & 06 & & 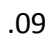 & & & & & & & & & & 10 \\
\hline$S_{-1}$ & & .02 & 02 & .02 & & & 55 & & 07 & .0 & 08 & & .0 & & & & & & 02 & & .07 \\
\hline$S_{0}$ & & .01 & לב & .04 & & דל & 04 & & .07 & .0 & .06 & .0 & .0 & .0 & & .0 & & & 03 & & .11 \\
\hline$S_{1}$ & 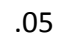 & .01 & .03 & .01 & .05 & .0 & .04 & .0 & .08 & .0 & .0 & .0 & .0 & .0 & & .0 & .0 & & .0 & & .05 \\
\hline$S_{2}$ & .06 & .00 & .02 & .03 & .0 & 0 & .05 & .0 & .05 & .0 & .06 & 0 & .0 & .0 & & .0 & & & .0 & & .0 \\
\hline$S_{3}$ & .11 & .02 & .03 & .03 & .03 & 0 & .03 & .0 & .06 & .0 & .05 & 0 & .0 & .09 & O & .06 & .06 & .04 & .02 & 0 & $.0 \varepsilon$ \\
\hline 4 & & .01 & .02 & .03 & & & .05 & & .06 & .0 & .0 & & & .0 & & & & & & & 05 \\
\hline & .05 & .03 & .02 & .02 & .0 & .03 & .05 & .0 & .07 & .0 & .10 & .0 & .1 & .0 & & .0 & .0 & & & & .0 \\
\hline & .04 & .01 & .02 & .01 & .0 & .0 & .05 & .03 & .10 & .1 & .08 & .0 & & .0 & & .0 & & & & & .07 \\
\hline$S_{7}$ & .06 & .01 & .03 & .03 & .03 & .04 & .03 & .02 & .06 & .0 & .07 & & .0 & .1 & & .0 & .03 & .02 & 03 & & .06 \\
\hline$S_{8}$ & .03 & .01 & .01 & .03 & .08 & .03 & .04 & .04 & .05 & .0 & .14 & .1 & .04 & .06 & & .09 & .08 & .01 & .00 & .01 & .06 \\
\hline$S_{9}$ & .07 & .03 & .02 & .02 & .03 & .00 & .02 & .03 & .16 & .08 & .05 & .0 & .10 & .08 & .0 & .02 & .02 & .05 & .03 & .02 & .07 \\
\hline & UY & 01 & .02 & .01 & .U3 & UL & US & 04 & I & Uל. & 04 & & US & .08 & US & . U6 & U3 & 03 & 04 & J3 & \\
\hline
\end{tabular}

Lastly, we calculated the probability of next day closing return state $p\left(x_{i}\right)$ by multiplying present return state and the conditional transition matrix. We calculated the expected closing return $\hat{R}_{t}$ for next day by using:

$$
\hat{R}_{t}=\sum_{i} x_{i} p\left(x_{i}\right)
$$

where $x_{i}$ denotes the middle points of the states for $i=-9, \ldots, 9$ and the boundaries of the states for $i=-10,10$.

\subsection{Methodology for Markov Chain with Fuzzy States}

Closing returns of the BIST 100 index are transformed into 21 fuzzy states from the high loss $S_{-10}$ to high return $S_{10}$ which is shown in Figure 1. We used triangular fuzzy numbers to obtain the membership degree of $R_{t}$ to the fuzzy states. 


\section{Figure 1: Fuzzy States for BIST 100 Index}

$\mu$

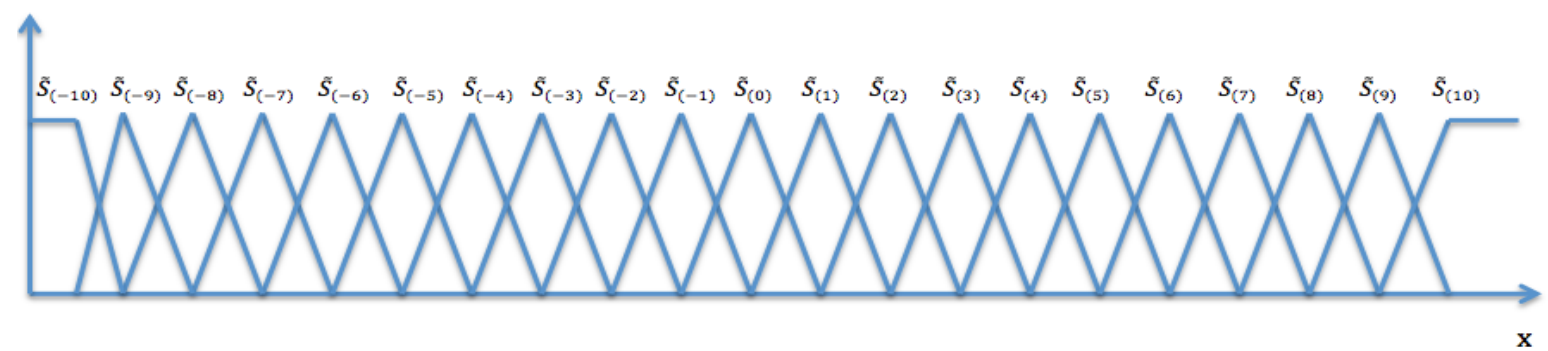

Let $\llbracket x \rrbracket$ be the greatest integer function and $R_{t}$ is the closing return of BIST 100 index. We define fuzzy state components of the returns $\tilde{S}_{i}$ and $\tilde{S}_{i+1}$ as following:

If $-2.25 \%<R_{t}<2.25 \%$ then $i=\llbracket \frac{R_{t}}{0.225} \rrbracket$ and

$$
\tilde{S}_{i}=\frac{(i+1)\left(0.225-R_{t}\right)}{0.225}, \tilde{S}_{i+1}=1-\tilde{S}_{i}
$$

If $R_{t} \leq-2.25 \%$ or $R_{t} \geq 2.25 \%$ then $\tilde{S}_{-10}=1$ or $\tilde{S}_{10}=1$ respectively.

Therefore $R_{t}$ numbers are transformed to the triangular fuzzy numbers for the time considered.

\section{Table 3: Transformed Fuzzy States of the Closing Returns for Some Days}

$\begin{array}{lllllllllllllllllllllll}\text { Date } & R_{t} & \tilde{S}_{-10} & \tilde{S}_{-9} & \tilde{S}_{-8} & \tilde{S}_{-7} & \tilde{S}_{-6} & \tilde{S}_{-5} & \tilde{S}_{-4} & \tilde{S}_{-3} & \tilde{S}_{-2} & \tilde{S}_{-1} & \tilde{S}_{0} & \tilde{S}_{1} & \tilde{S}_{2} & \tilde{S}_{3} & \tilde{S}_{4} & \tilde{S}_{5} & \tilde{S}_{6} & \tilde{S}_{7} & \tilde{S}_{8} & \tilde{S}_{9} & \tilde{S}_{10}\end{array}$ \begin{tabular}{ll|lllllllllllllllllllll} 
Mar.10, 2016 & $0.36 \%$ & .00 & .00 & .00 & .00 & .00 & .00 & .00 & .00 & .00 & .00 & .00 & .40 & .60 & .00 & .00 & .00 & .00 & .00 & .00 & .00 & .00
\end{tabular} \begin{tabular}{ll|lllllllllllllllllllll} 
Mar.09, 2016 & $1.36 \%$ & .00 & .00 & .00 & .00 & .00 & .00 & .00 & .00 & .00 & .00 & .00 & .00 & .00 & .00 & .00 & .00 & .96 & .04 & .00 & .00 & .00
\end{tabular}

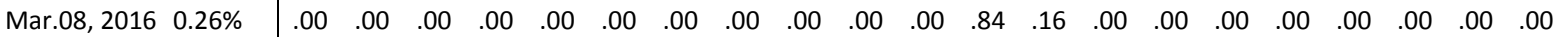
\begin{tabular}{ll|lllllllllllllllllllll} 
Mar.07, 2016 & $0.38 \%$ & .00 & .00 & .00 & .00 & .00 & .00 & .00 & .00 & .00 & .00 & .00 & .31 & .69 & .00 & .00 & .00 & .00 & .00 & .00 & .00 & .00
\end{tabular} \begin{tabular}{ll|lllllllllllllllllllll} 
Mar.06, 2016 & $0.47 \%$ & .00 & .00 & .00 & .00 & .00 & .00 & .00 & .00 & .00 & .00 & .00 & .00 & .91 & .09 & .00 & .00 & .00 & .00 & .00 & .00 & .00
\end{tabular}

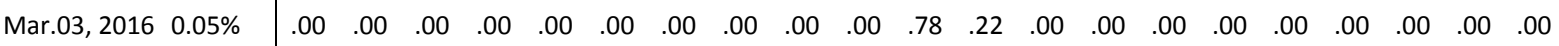
\begin{tabular}{ll|lllllllllllllllllllll} 
Mar.02, 2016 & $1.10 \%$ & .00 & .00 & .00 & .00 & .00 & .00 & .00 & .00 & .00 & .00 & .00 & .00 & .00 & .00 & .11 & .89 & .00 & .00 & .00 & .00 & .00
\end{tabular}

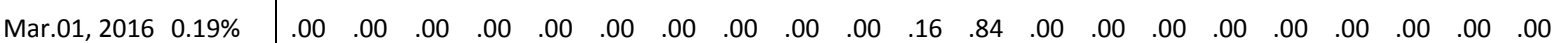
Feb.29, 2016 1.18\% $\quad \begin{array}{llllllllllllllllllllll}.00 & .00 & .00 & .00 & .00 & .00 & .00 & .00 & .00 & .00 & .00 & .00 & .00 & .00 & .00 & .76 & .24 & .00 & .00 & .00 & .00\end{array}$

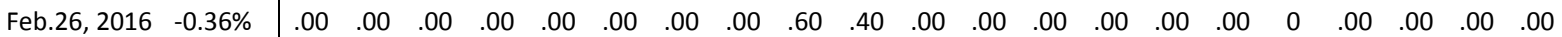

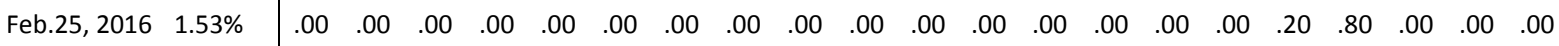
\begin{tabular}{ll|llllllllllllllllllllllll} 
Feb.24, 2016 & $-1.90 \%$ & .00 & .44 & .56 & .00 & .00 & .00 & .00 & .00 & .00 & .00 & .00 & .00 & .00 & .00 & .00 & .00 & .00 & .00 & .00 & .00 & .00
\end{tabular}

Within this framework, MCFS which depend on the fuzzy set theory provides more realistic description to the problems than the MC. Then we calculated the fuzzy transition probability by using the conditional probability of the fuzzy state $\tilde{S}_{j}$, given the fuzzy state $\tilde{S}_{i}$. With this way, we replaced fuzzy transition probability with crisp transition probability. Then we obtained the probabilistic transition matrix of the fuzzy states by using equations (7) and (8). 
Table 4: Probabilistic Transition Matrix of the Fuzzy States

\begin{tabular}{|c|c|c|c|c|c|c|c|c|c|c|c|c|c|c|c|c|c|c|c|c|c|}
\hline & $\tilde{S}_{-10}$ & -9 & $\tilde{S}_{-8}$ & $\tilde{S}_{-7}$ & $\tilde{S}_{-6}$ & $\tilde{S}_{-5}$ & $\tilde{S}_{-4}$ & $\tilde{S}_{-3}$ & $\tilde{S}_{-2}$ & $\tilde{S}_{-1}$ & $S_{0}$ & $\tilde{S}_{1}$ & $\tilde{S}_{2}$ & $\tilde{S}_{3}$ & $\tilde{S}_{4}$ & $\tilde{S}_{5}$ & $\tilde{S}_{6}$ & $S_{7}$ & $S_{8}$ & & $\tilde{S}_{10}$ \\
\hline & .17 & .03 & .03 & .04 & .03 & .02 & .02 & .04 & .03 & .04 & .05 & .04 & .05 & .05 & .05 & .03 & .03 & .04 & .03 & .03 & \\
\hline & .12 & .02 & .03 & .05 & .08 & .02 & .06 & .05 & .02 & .05 & 04 & .06 & .04 & .03 & .04 & .04 & .05 & .04 & .00 & & \\
\hline & .07 & .00 & .01 & .04 & .04 & .02 & .02 & .05 & .05 & .08 & .05 & .09 & .08 & .04 & 06 & .07 & .06 & .03 & .02 & & 10 \\
\hline & .09 & .02 & .02 & .03 & .05 & .04 & .02 & .05 & .07 & .04 & 03 & .07 & .05 & .04 & .04 & .04 & .05 & .04 & .04 & 03 & .13 \\
\hline & .10 & .02 & .03 & .04 & .02 & .02 & .04 & .07 & .05 & .05 & .04 & .06 & .05 & .06 & .05 & .04 & .07 & .04 & .03 & 02 & .10 \\
\hline & .07 & .02 & .03 & .03 & .02 & .02 & .04 & .06 & .08 & .07 & .04 & .05 & .07 & .05 & .07 & .05 & .07 & .03 & .03 & .02 & .12 \\
\hline$\tilde{S}_{-4}$ & .08 & .04 & .03 & .02 & .05 & .04 & .04 & .06 & .05 & .06 & .08 & .06 & .07 & .04 & .05 & .04 & .05 & .03 & .02 & 01 & .06 \\
\hline & .09 & .03 & .02 & .01 & .03 & .04 & .04 & .06 & .04 & .07 & .08 & .08 & .07 & .06 & .04 & .03 & .04 & .04 & .02 & .02 & .08 \\
\hline$\tilde{S}_{-2}$ & .06 & .02 & .03 & .02 & .03 & .05 & .05 & .05 & .05 & .04 & .07 & .06 & .06 & .07 & .05 & .04 & .06 & .03 & .02 & .03 & .10 \\
\hline$\tilde{S}_{-1}$ & .07 & .02 & .02 & .03 & .03 & .04 & .04 & .05 & .07 & .05 & .06 & .07 & .07 & .05 & .05 & .04 & .04 & .05 & .03 & .03 & .09 \\
\hline$\tilde{S}_{0}$ & .06 & .02 & .03 & .04 & .05 & .05 & .04 & .04 & .06 & .08 & .06 & .07 & .04 & .04 & .05 & .03 & .04 & .03 & .04 & .02 & .11 \\
\hline$\tilde{\tilde{\sigma}}$ & .06 & .03 & .02 & .03 & .05 & .06 & .04 & .04 & .07 & .06 & .07 & .08 & .06 & .06 & .06 & .04 & .04 & .02 & .03 & .02 & .08 \\
\hline$\tilde{S}_{2}$ & .06 & .02 & .02 & .02 & .03 & .04 & .05 & .05 & .05 & .05 & .06 & .07 & .07 & .06 & .06 & .04 & .05 & .05 & .04 & .02 & .07 \\
\hline$\tilde{\kappa}$ & .09 & .01 & .03 & .02 & .03 & .03 & .03 & .04 & .07 & .08 & .05 & .0 & .06 & .07 & .06 & .05 & .05 & .05 & .03 & .01 & .09 \\
\hline & .10 & .02 & .03 & .03 & .04 & .04 & .05 & .05 & .05 & .06 & .07 & .06 & .07 & .05 & .04 & .06 & .05 & .04 & .02 & .01 & .06 \\
\hline$\tilde{S}_{5}$ & .07 & .01 & .02 & .03 & .05 & .06 & .05 & .05 & .05 & .06 & .09 & .08 & .09 & .04 & .02 & .07 & .04 & .03 & .02 & .02 & .05 \\
\hline$\tilde{S}_{6}$ & .08 & .01 & .02 & .03 & .04 & .03 & .04 & .06 & .07 & .08 & .11 & .07 & .08 & .04 & .03 & .05 & .03 & .03 & .03 & .01 & .06 \\
\hline$\tilde{\boldsymbol{c}}$ & .05 & .03 & .03 & .02 & .0 & .03 & .04 & .05 & .07 & .09 & .06 & .05 & .06 & .10 & .06 & .03 & .02 & .02 & .03 & .02 & .10 \\
\hline & .03 & .02 & .02 & .03 & .05 & .03 & .02 & .03 & .05 & .06 & .09 & .1 & .09 & 10 & .08 & .04 & .05 & .03 & .02 & .01 & .06 \\
\hline$\tilde{\tilde{c}}$ & .06 & .02 & .01 & .04 & .04 & .01 & .02 & .03 & .08 & .09 & .11 & .08 & .05 & .06 & .05 & .05 & .06 & .02 & .02 & .01 & .09. \\
\hline$\tilde{\boldsymbol{c}}$ & .10 & .02 & .01 & .02 & .03 & .02 & .04 & .05 & .07 & .05 & .04 & .07 & .06 & .07 & .05 & .05 & .04 & .03 & .03 & .03 & .14 \\
\hline
\end{tabular}

In Table 4, the closing returns are considered as a stochastic process with 21 fuzzy state space $\left\{\tilde{S}_{-10}, \ldots, \tilde{S}_{10}\right\}$ with Markov chain structure. The conditional partition degree of passing from state $\tilde{S}_{5}$ to $\tilde{S}_{2}$ is $\widetilde{P}\left(\tilde{S}_{2} \mid \tilde{S}_{5}\right)=9 \%$.

Lastly, we calculated next day closing returns partition degrees to the states $\tilde{p}\left(x_{i}\right)$, by multipliying present return partitions and the conditional transition matrix of the fuzzy states. And we calculated the expected closing return $\hat{R}_{t}$ for next day by using equation (9).

$$
\hat{R}_{t}=\sum_{i} x_{i} \tilde{p}\left(x_{i}\right)
$$

Categories of the states and the conditional transition probability matrices are formulated and calculated in Excel by using Excel IF function.

\section{FINDINGS AND DISCUSSIONS}

The MCFS model gives information about risky days. When a daily return substantially increased $(2,25 \%$ or greater), the next day's return also substantially increased or decreased with the probability of $14 \%$ or $10 \%$ respectively. On the other hand, when a daily return substantially decreased $(2,25 \%$ or greater), the next day's return also substantially increased or decreased with the probability of $15 \%$ or $17 \%$ respectively. This result is not significiantly different from that of the MC model in Table 2.

\section{Table 5: Estimated Closing Return $\widehat{R}_{t}$ with MC Model for Feb.12, 2016}

$$
\begin{aligned}
& \begin{array}{lllllllllllllllllllll}
S_{-10} & S_{-9} & S_{-8} & S_{-7} & S_{-6} & S_{-5} & S_{-4} & S_{-3} & S_{-2} & S_{-1} & S_{0} & S_{1} & S_{2} & S_{3} & S_{4} & S_{5} & S_{6} & S_{7} & S_{8} & S_{9} & S_{10}
\end{array} \\
& p\left(x_{i}\right)\left|\begin{array}{llllllllllllllllllllllll}
0.05 & 0.03 & 0.04 & 0.03 & 0.07 & 0.03 & 0.02 & 0.08 & 0.08 & 0.05 & 0.07 & 0.06 & 0.07 & 0.04 & 0.07 & 0.05 & 0.03 & 0.02 & 0.01 & 0.01 & 0.06
\end{array}\right| \\
& x_{i} \quad-2.40-2.16-1.92-1.68-1.44-1.20-0.96-0.72-0.48-0.240 .000 .240 .48 \quad 0.720 .961 .201 .441 .681 .92 \quad 2.162 .40 \\
& \hat{R}_{t}=-0.12 \%
\end{aligned}
$$

In Table 5, we have shown the probability of closing return $p\left(x_{i}\right)$ on Feb.12, 2016 with MC model and we computed the expected return $\hat{R}_{t}$. 
Table 6: Estimated closing return $\widehat{R}_{t}$ with MCFS model for Feb.12, 2016

$$
\begin{array}{c|ccccccccccccccccccccc} 
& \tilde{S}_{-10} & \tilde{S}_{-9} & \tilde{S}_{-8} & \tilde{S}_{-7} & \tilde{S}_{-6} & \tilde{S}_{-5} & \tilde{S}_{-4} & \tilde{S}_{-3} & \tilde{S}_{-2} & \tilde{S}_{-1} & \tilde{S}_{0} & \tilde{S}_{1} & \tilde{S}_{2} & \tilde{S}_{3} & \tilde{S}_{4} & \tilde{S}_{5} & \tilde{S}_{6} & \tilde{S}_{7} & \tilde{S}_{8} & \tilde{S}_{9} & \tilde{S}_{10} \\
\tilde{p}\left(x_{i}\right) & 0.08 & 0.03 & 0.03 & 0.02 & 0.05 & 0.03 & 0.04 & 0.06 & 0.06 & 0.07 & 0.07 & 0.06 & 0.07 & 0.04 & 0.06 & 0.04 & 0.05 & 0.03 & 0.02 & 0.01 & 0.07 \\
x_{i} & -2.25 & -2.03 & -1.80 & -1.58 & -1.35 & -1.13 & -0.90 & -0.68 & -0.45 & -0.23 & 0.00 & 0.23 & 0.45 & 0.68 & 0.90 & 1.13 & 1.35 & 1.58 & 1.80 & 2.03 & 2.25
\end{array}
$$

$\hat{R}_{t}=-0.04 \%$ and the actual closing return $R_{t}=-0.01 \%$ on Feb.12, 2016 .

In Table 6, we have shown the probability of closing return $\tilde{p}\left(x_{i}\right)$ on Feb.12, 2016 with MCFS model and we computed the expected return $\hat{R}_{t}$.

Table 7: Estimated $\boldsymbol{R}_{\boldsymbol{t}}$ for Some Days

\begin{tabular}{|l|c|c|c|}
\hline Dates & $\widehat{\boldsymbol{R}}_{\boldsymbol{t}}$ with MC Model & $\widehat{\boldsymbol{R}}_{\boldsymbol{t}}$ with MCFS Model & Actual $\left(\boldsymbol{R}_{\boldsymbol{t}}\right)$ \\
\hline Feb.12, 2016 & $-0.12 \%$ & $-0.04 \%$ & $-0.01 \%$ \\
\hline Jan.15, 2016 & $-0.08 \%$ & $-0.06 \%$ & $-0.52 \%$ \\
\hline Nov.11, 2015 & $-0.10 \%$ & $0.15 \%$ & $0.67 \%$ \\
\hline Nov.15, 2016 & $-0.08 \%$ & $0.05 \%$ & $0.98 \%$ \\
\hline MAE & $0.60 \%$ & $0.49 \%$ & \\
\hline
\end{tabular}

In Table 7, we have shown some estimation results for some days which are choosen randomly. And we used the mean absolute error MAE to measure how our prediction is close to the eventual outcomes.

$$
M A E=\frac{1}{n} \sum_{i=1}^{n}\left|e_{i}\right|
$$

where $e_{i}$ denotes the error. From Table 1 , one can see that MCFS model is better than MC model for forecasting the stock market index.

\section{Validation test for the Markov model with fuzzy states}

To validate MCFS, a comparison is carried out between the expected and actual values of the BIST 100 returns. The test of $\chi_{(0.05)}^{2}$ was applied between expected and actual values to evaluate the $95 \%$ significance of the results. Test values have been calculated by

$$
\chi_{(0.05)}^{2}=\sum_{i=1}^{21} \frac{\left(A V_{i}-E V_{i}\right)^{2}}{E V_{i}}
$$

where $E V_{i}$ and $A V_{i}$ are the values estimated by the model and the actual values, respectively For each sample, the value of

\begin{tabular}{|c|c|c|c|c|c|c|c|c|c|c|c|c|c|c|c|c|c|c|c|}
\hline & $\tilde{S}_{-10} \tilde{S}_{-9}$ & $\tilde{S}_{-8}$ & $\tilde{S}_{-7}$ & $\tilde{S}_{-6}$ & $\tilde{S}_{-5}$ & $\tilde{S}_{-4}$ & $\tilde{S}_{-3}$ & $\tilde{S}_{-2} \tilde{S}_{-1}$ & $\tilde{S}_{0}$ & $\tilde{S}_{1}$ & $\tilde{S}_{2}$ & $\tilde{S}_{3}$ & $\tilde{S}_{4}$ & $\tilde{S}_{5}$ & $\tilde{S}_{6}$ & $\tilde{S}_{7}$ & $\tilde{S}_{8}$ & $\tilde{S}_{9}$ & $\tilde{S}_{10}$ \\
\hline$\tilde{p}\left(x_{i}\right)$ & .08 & .03 & .02 & .05 & .03 & .04 & .06 & $.06 \quad .07$ & .07 & .06 & .07 & .04 & .06 & .04 & .05 & .03 & .02 & .01 & .07 \\
\hline $\begin{array}{c}\text { Actual } \\
R_{t}\end{array}$ & $.00 \quad .00$ & .00 & .00 & .00 & .00 & .00 & .00 & $.00 \quad .04$ & .96 & .00 & .00 & .00 & .00 & .00 & .00 & .00 & .00 & .00 & .00 \\
\hline
\end{tabular}
the chi-square $\left(\chi^{2}\right)$ statistic is calculated based on the null hypothesis $H_{0}$. In this study, the degree of freedom is 20 (21 categories-1). The critical value (CV) in the test with $95 \%$ significance is 31.41 .

Table 8: Partition degrees of estimated closing returns $\widehat{R}_{t}$ and the actual return $R_{t}$ for Feb.12,2016 and Nov.11,2015

Date Feb.12,2016

Date Nov.11,2015

$\begin{array}{lllllllllllllllllllll}\tilde{S}_{-10} & \tilde{S}_{-9} & \tilde{S}_{-8} & \tilde{S}_{-7} & \tilde{S}_{-6} & \tilde{S}_{-5} & \tilde{S}_{-4} & \tilde{S}_{-3} & \tilde{S}_{-2} & \tilde{S}_{-1} & \tilde{S}_{0} & \tilde{S}_{1} & \tilde{S}_{2} & \tilde{S}_{3} & \tilde{S}_{4} & \tilde{S}_{5} & \tilde{S}_{6} & \tilde{S}_{7} & \tilde{S}_{8} & \tilde{S}_{9} & \tilde{S}_{10}\end{array}$ $\begin{array}{llllllllllllllllllllll}\tilde{p}\left(x_{i}\right) & .07 & .02 & .03 & .03 & .03 & .02 & .04 & .06 & .07 & .07 & .05 & .05 & .07 & .04 & .07 & .04 & .07 & .03 & .02 & .01 & .11\end{array}$ $\begin{array}{llllllllllllllllllllllll}\text { Actual } & 0.67 \% & .00 & .00 & .00 & .00 & .00 & .00 & .00 & .00 & .00 & .00 & .00 & .00 & .02 & .98 & .00 & .00 & .00 & .00 & .00 & .00 & .00\end{array}$ 
The values of the $\chi_{(0.05)}^{2}$ test are 11.8 on Feb.12, 2016 and 20.3 on Nov.11,2015 which are lower than 31.41 and also $\chi_{(0.05)}^{2}$ test values are lower than $\mathbf{3 1 . 4 1}$ for each sample. The results show that the MCFS model with the triangular fuzzy numbers is plausible.

\section{CONCLUSION}

We have predicted behaviour of the BIST 100 stock return index for next day using four days. Using the MC and MCFS models, forecasts of the daily stock returns are compared. The results give sensitive and significant information to the investors about investment opportunities of the BIST 100 index for the daily buying and selling strategies when the present return is known.

In risky days, when a daily return substantially increased or decreased, the next day's return also substantially increased or decreased for both models. The transition probabilities of daily returns in non-risky days would be significiantly lower than those in risky days for both models.

The MCFS model can be used to forecast the returns for smaller time (less than one day) intervals which may give more investment opportunities. Inverstors can earn higher than the average return in risky days in a short run. Besides the MCFS model can be useful for estimating returns of individual common stocks and also for the other investment instruments. Finally, MCFS provided a higher forecasting accuracy to the investors compared to the MC model.

\section{REFERENCES}

Avrachenkov K.E. \& Sanchez E. 2000, "Fuzzy Markov chains", IPMU, Spain, pp. 1851-1856.

Badge, J. 2012, "Forecasting of Indian Stock Market by Effective Macro- Economic Factors and Stochastic Model", Journal of Statistical and Econometric Methods, vol. 1 (2), pp. 39-51, ISSN: 2241-0384 (print), 2241-0376 (online) Sciencepress Ltd.

Bellman, R. 1957, "A Markov Decision Process", Journal of Mathematics and Mechanics 6.

Box G.E.P., Jenkins, G. M. 1976, "Time series analysis: forecasting and control", San Fransisco, CA: Holden-Day.

Chiang W.C., Urban T. L. \& Baldridge, G.W. 1996, "A neural network approach to mutual fund net asset value forecasting", Omega International Journal of Management Science, vol. 24 (2), pp. 205-215.

Gupta A. \& Dhingra B. 2012, "Stock Market Prediction Using Hidden Markov Models", Non-Student members, IEEE.

Hassan, Md. R. \& Nath, B. 2005, "Stock Market forecasting using Hidden Markov Model: A New Approach", Proceeding of the $5^{\text {th }}$ international conference on intelligent Systems Design and Application 0-7695-2286-06/05, IEEE.

Hassan, Md. R., Nath, B. \& Kirley, M. 2006, "HMM based Fuzzy Model for Time Series Prediction", IEEE International Conference on Fuzzy Systems, pp. 2120-2126.

Hassan, Md. R., Nath, B. \& Kirley, M. 2007, "A fusion model of HMM, ANN and GA for stock market forecasting", Expert systems with Applications, pp. 171-180.

Henry, M. K. M. 1993, "Causality of interest rate, exchange rate and stock prices at stock market open and close in Hong Kong", Asia Pacific Journal of Management, vol. 10 (2), pp. 123-143.

Kim, K.J. \& Han, I. 2000, "Genetic algorithms approach to feature discretization in artificial neural networks for the prediction of stock price index", Expert Systems with Applications, vol.19, pp.125-132.

Kruce, R., Buck- Emden, R. \& Cordes, R. 1987, "Process or Power Considerations-An Application to Fuzzy Markov Chains", Fuzzy Sets and Systems, pp. 289-299.

Kuranoa, M., Yasuda, M., Jakagami, J. \& Yoshida, Y. 2006, "A Fuzzy Approach to Markov Decision Processes with Unceratin Transition Probabilities", Fuzzy Sets and Systems, 157, pp. 2674-2682.

Pardo, M.J. \& Fuente, D. 2010, "Fuzzy Markovian Decision Processes: Application to Queueing Systems", Computers and Mathematics with Applications, 60, pp. 2526-2535.

Rabiner, L.R. 1993, "A tutorial on HMM and Selected Applications in Speech Recognition", In: [WL], proceedings of the IEEE, vol. 77 (2), pp. 267- 296.

Rabiner, L.R., Juang, B. 1993, "Fundamentals of Speech Recognition", Prentice-Hall, Englewood Cliffs, NJ.

Romahi Y. \& Shen, Q. 2000, "Dynamic financial forecasting with automatically induced fuzzy associations", In Proceedings of the 9th international conference on fuzzy systems, pp. 493-498.

Salzenstein, F., Collet, C., Lecam, S. \& Hatt, M. 2007, "Non-Stationary Fuzzy Markov Chain", Pattern Recognition Letters, 28, pp. 2201-2208. 
Sanchez, E. 1976, "Resolution of Composite Fuzzy Relation Equations", Information and Control, 30, pp. 38-48.

Stow'ınski, R. (ed.) 1998, "Fuzzy Sets in Decision Analysis", Operation Research and Statistics, Kluwer Academic Publishers.

Symeonaki, M.A. \& Stamou, G.B. 2004, "Theory of Markov Systems with Fuzzy States", Fuzzy Sets and Systems, 143, pp. 427-445.

Thomason M. 1977, "Convergence of Powers of a Fuzzy Matrix", Journal of Mathematical Analysis and Applications, 57, pp. 476-480.

Vajargah, B.F. \& Gharehdaghi, M. 2012, "Ergodicity of fuzzy Makov chains based on simulation using Halton sequences", The Journal of Mathematics and Computer Science, vol. 4, no. 3, pp. 380-385.

White, H., 1988, "Economic prediction using neural networks: the case of IBM daily stock returns", Department of Economics, University of California, San Diego.

White, H. 1988, "Economic prediction using neural networks: the case of IBM daily stock returns", In Proceedings of the second IEEE annual conference on neural networks, II, pp. 451-458.

White, H. 1989, "Learning in artificial neural networks: a statistical perspective", Neural Computation, vol. 1, pp. 425-464.

Yoshida, Y. 1994, "Markov chains with a transition possibility measure and fuzzy dynamic programming", Fuzzy Sets and Systems, 66, pp. 39-57.

Zadeh, L.A. 1965, "Fuzzy Sets", Information Control, 8, pp. 338-353.

Zhou, X., Tang, Y., Xie, Y., Li, Y. \& Zhang, Y. 2013, "A Fuzzy Probability- based Markov Chain Model for Electric Power Demand Forecasting of Beijing", Energy and Power Engineering, China, pp. 488-492.

Borsa Istanbul, http://www.borsaistanbul.com 